\title{
Nuevas conflictividades y vínculos entre movimientos sociales, partidos políticos y gobierno en el Uruguay progresista (2005-2020)
}

\author{
Germán Bidegain* \\ Martín Freigedo* \\ Diego Puntigliano Casulo*
}

\section{Resumen}

En 2005, el triunfo del Frente Amplio (FA) modificó la estructura de oportunidad política del país por tratarse de un partido político de izquierda, tradicional aliado de los movimientos sociales en las distintas campañas de protesta contra los gobiernos que se sucedieron entre 1985 y 2014. Luego de 15 años de gobierno del FA, cabe preguntarse: ¿Qué novedades existieron en la matriz de articulación sociopolítica del país? ¿Cómo afectaron los cambios a la conflictividad sociopolítica del país? En este artículo nos centraremos en tres expresiones de conflictividad novedosas que dieron cuenta de patrones de relacionamiento inéditos entre movimientos sociales, sistema político y gobierno: la ola de protesta del movimiento ambiental contra inversiones extranjeras directas, los grupos anti-derechos que reaccionaron a la nueva agenda de derechos, y el movimiento Un Solo Uruguay, que planteó conflictos redistributivos asociados a las demandas tradicionales de las gremiales rurales. Estos casos expresan dos novedades importantes. Por un lado, rompen con la histórica articulación entre el FA y los movimientos sociales. Por otro, el surgimiento de movimientos sociales asociados a las demandas de los partidos de derecha.

Palabras clave: Uruguay, Frente Amplio, movimientos sociales, conflictos sociales

* Universidad de la República de Uruguay, Montevideo, Uruguay. 


\section{New contentiousness and links between social movements, political parties and the government in progressive Uruguay (2005-2020)}

\section{Abstract}

In 2005, the electoral victory of the Frente Amplio (FA) changed the Uruguayan political opportunity structure. This left-wing party was an ally to the country's social movements in several campaigns against right-wing ruling governments between 1985 and 2004. The FA remained in power for 15 years. What were the changes in the country's historical articulation pattern between social and political actors? How did these changes affect the country's sociopolitical conflicts? In this article we will focus on three emerging expressions of conflict that accounted for unprecedented relationship patterns between social movements, the political system and the government: the protesting wave of environmental movement against direct foreign investment, anti-rights groups' reaction to the new rights agenda, and the movement Un Solo Uruguay, which raised redistributive issues in connection with rural unions' traditional demands. These cases explore two important breakthroughs. On the one hand, they effectively break with the historical articulation between FA and the social movements. On the other, the rise of social movements linked to right-wing party claims.

Keywords: Uruguay, Frente Amplio, social movements, social conflicts

\section{Introducción}

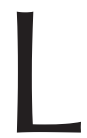

a llegada al gobierno por primera vez de un partido de izquierda, el Frente Amplio (FA), inició un ciclo de tres gobiernos progresistas (2005-2020) en Uruguay. Anteriormente, como oposición desde la transición democrática de 1985, este partido desarrolló fuertes lazos con los distintos movimientos sociales para contrarrestar diversas iniciativas de los sucesivos gobiernos de los partidos tradicionales, sobre todo a la agenda neoliberal de la década de los 90'. El uso reiterado de mecanismos de democracia directa para ejercer su rol de oposición es una peculiaridad del caso uruguayo, con resultados importantes para frenar esta agenda (Bidegain; Tricot, 2017; Monestier, 2010; Moreira, 2004). 
El triunfo del FA implicó una situación inédita en el país, que modificó sustantivamente la estructura de oportunidad política, generando expectativas en sus votantes y en las organizaciones sociales de izquierda, debido a la existencia de una ambiciosa agenda de reformas que había cimentado anteriormente. Como veremos, también generó reacciones de grupos opuestos al programa frenteamplista. Teniendo en cuenta el nuevo escenario político, este trabajo se pregunta: ¿Qué novedades existieron en la matriz de articulación sociopolítica del país? ¿Cómo afectaron los cambios a la conflictividad sociopolítica del país?

Por una parte, el nuevo contexto político generó una estructura de oportunidad política (EOP) más abierta para los movimientos sociales tradicionalmente aliados al FA. Esto implicó una serie de reformas que atendió distintos reclamos históricos, e incluso la incorporación de algunos cuadros sociales a la estructura estatal en una lógica de activismo burocrático (Abers; Von Bülow, 2011). Lo anterior no significó la cooptación de los movimientos por parte de los gobiernos - aquellos mantuvieron ciertos grados de conflictividad para presionar a las autoridades a tomar en cuenta sus reclamos (en temas tan diversos como política educativa, pensiones, derechos humanos o cooperativismo de vivienda, por ejemplo). De todas formas, primó una tónica general de menor confrontación de estos movimientos, en comparación con los gobiernos anteriores de derecha. En este sentido, más allá de tensiones concretas, se ha postulado la existencia de un "consenso progresista" que pautó las relaciones entre el gobierno y las organizaciones sociales del campo popular (Falero; Fry, 2017).

Por otra parte, las novedades más interesantes en relación a la conflictividad durante estos quince años provinieron de la manifestación de nuevos o reconfigurados actores colectivos con agendas opuestas a las políticas del FA en temas diversos. En este trabajo nos centramos en tres expresiones de conflictividad que dan cuenta de una rearticulación en la matriz de intermediación sociopolítica durante los gobiernos del FA, a partir del vínculo específico entre movimientos sociales, partidos políticos y gobierno. En primer lugar, analizamos la acción colectiva de distintos 
grupos conservadores, que buscaron oponerse a los avances de la "nueva agenda de derechos" (NAD) impulsada desde los gobiernos frenteamplistas, así como impulsar una agenda política de corte represivo para combatir la inseguridad. En segundo lugar, tratamos el fortalecimiento del movimiento ambientalista, que logró una gran capacidad de movilización y desarrolló una mirada crítica de todo el sistema de partidos, incluido el FA. En tercer lugar, el surgimiento del movimiento Un Solo Uruguay. Este movimiento inédito, de corte rural, retomó algunas demandas históricas de las cámaras empresariales y agrarias, pero desarrolló un repertorio de acción característico de movimientos sociales. ${ }^{1}$

A través de estos casos se dará cuenta de la forma de rearticulación entre actores colectivos y partidos políticos, mostrando dos novedades: i) movimientos que rompen con la histórica articulación entre el FA y la izquierda social, y ii) el surgimiento de movimientos que comparten demandas con los partidos de derecha. A estas novedades, que expresan cambios en los patrones internos de intermediación sociopolítica, se puede agregar otra desde una mirada regional: los conflictos ambientales y la reacción conservadora frente a la nueva agenda de derechos acerca a Uruguay a dinámicas de conflicto latinoamericanas.

El trabajo se basa en revisión bibliográfica, de fuentes secundarias y entrevistas de nuestras agendas de investigación personales sobre protesta y acción colectiva en Uruguay. En la sección siguiente proponemos un marco teórico y contextual para dar cuenta de algunos rasgos generales de la conflictividad histórica uruguaya, y presentar algunos conceptos

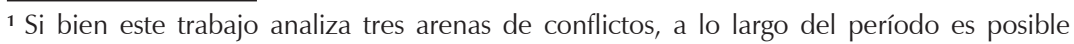
encontrar otros asuntos que han llevado a disputas en torno a la agenda política del FA. El ejemplo más importante es probablemente la disputa en torno a la agenda de seguridad ciudadana, con un fuerte impacto en la opinión pública debido a la promoción por parte de algunas fracciones de partidos políticos de derecha de dos plebiscitos (en 2014 y 2019). En este trabajo no se abordarán estos casos debido a que el impulso de los plebiscitos se dio por la iniciativa de fracciones de partidos políticos, con una participación marginal de actores sociales. La movilización social se dio en contra de las iniciativas, replicando las experiencias clásicas de alianza entre organizaciones sociales (sindicales, de diversidad, feministas, estudiantiles etc.) y el FA. 
relevantes para el estudio de los cambios acontecidos en los últimos años. Esta sección introduce también cómo se rearticuló la relación entre el FA y sus aliados históricos del campo social. Las tres secciones siguientes presentan los aspectos principales de los casos de estudio en que centramos nuestro análisis. Finalmente, presentamos una sección de consideraciones finales que sintetiza los hallazgos principales del trabajo.

\section{Marco teórico y contextual: Estado, gobierno, partidos y movimientos sociales en Uruguay}

Vale la pena comenzar esta sección explicitando la definición de movimientos sociales que adoptamos en trabajo. En este respecto, seguimos la propuesta de Della Porta y Diani (2011, p. 43), que los definen como "procesos sociales diferenciados consistentes en mecanismos a través de los cuales actores comprometidos en la acción colectiva: - se involucran en relaciones conflictivas con oponentes claramente identificados; - se vinculan en densas redes informales; y - comparten una identidad diferenciada."

En el estudio sobre estos fenómenos, los vínculos entre movimientos sociales, Estado, gobierno y partidos políticos han ocupado un espacio relevante en la reflexión teórica y la investigación empírica de la literatura. Más allá de la relevancia histórica de las variables político-institucionales en la corriente del proceso político (McAdam, 1999; McAdam et al., 1996; Tilly, 1978), desde comienzos del siglo XXI ha ganado fuerza la preocupación específica por analizar las fronteras/puentes existentes entre la política institucional y no institucional (Goldstone, 2003; Von Bülow; Bidegain, 2017).

En el centro de los distintos enfoques que se han desarrollado, existen posturas teóricas diferentes sobre las fronteras entre la acción política institucional y no institucional, con consecuencias analíticas relevantes, ya que estos debates afectan la propia definición del objeto de estudio "movimiento social". En este sentido, Abers y Von Bülow (2011) identificaron dos corrientes analíticas que, a partir de los años 2000, buscaron ampliar 
las fronteras de estudio de movimientos sociales. Por una parte, la agenda propuesta en Dynamics of contention (McAdam et al., 2001), que buscó ampliar la mirada de "contienda política" a otros actores y casos de protesta. Por otra parte, una agenda orientada a incorporar como fenómeno de estudio las acciones no contenciosas de los movimientos sociales, prestando especial atención a repertorios no conflictivos como el "activismo burocrático" al interior del Estado (Abers, 2019; Silva; Oliveira, 2011).

Las variables políticas tienen particular relevancia en el estudio del caso uruguayo debido a su histórica matriz estadocéntrica y partidocrática (Chasquetti; Buquet, 2004; Filgueira, F. et al., 2003). Aunque a fines de la dictadura (1973-1985) los movimientos sociales ganaron cierta preponderancia a causa de la proscripción de los partidos, una vez recuperada la democracia los partidos ocuparon nuevamente el centro de la escena (Filgueira, C. H., 1985). Esto no significa que los actores sociales no hayan tenido un rol importante en el devenir político del país. Muy por el contrario, los movimientos sociales uruguayos lideraron entre 1985 y 2005 distintas campañas que afectaron el marco legal del país en temas centrales como la privatización de las empresas públicas, el sistema de pensiones o el derecho al agua. Sin embargo, y a diferencia de la protesta en otros países de la región (Joignant et al., 2017), buena parte de las campañas de los movimientos sociales uruguayos se canalizaron a través del uso intenso de mecanismos de democracia directa (Altman, 2011; Bidegain; Tricot, 2017). Entre 1985 y 2005 se dio una dinámica de intermediación sociopolítica en que distintos movimientos sociales (trabajadores, cooperativistas, estudiantes etc.) trabajaron con el FA para oponerse a la agenda neoliberal impulsada por los sucesivos gobiernos del país. Estas acciones mantuvieron el rol central histórico del Estado a pesar de los embates pro mercado de los distintos gobiernos (Monestier, 2010; Moreira, 2004).

Michael Hanagan (1998, p. 5-6) ofrece una clasificación de utilidad para caracterizar los vínculos entre partidos y movimientos sociales. De acuerdo a su propuesta, existen cinco tipos ideales de relacionamiento entre 
estos actores. En primer lugar, la articulación ocurre cuando un movimiento social se estructura en torno a un partido político, siendo funcional a los intereses del último. En segundo lugar, la permeabilidad refiere a una relación en la que las organizaciones de movimientos sociales operan dentro de un partido para influenciar su toma de postura. Supone mucha cercanía y lealtad de parte de los activistas sociales al partido, del cual son militantes y que esperan Ilevar hacia las posiciones del movimiento. En tercer lugar, Hanagan identifica a la alianza como aquella relación en que las organizaciones de movimientos sociales desarrollan alianzas ad hoc con partidos o fracciones de partidos para llevar adelante objetivos comunes. En este caso, partidos y movimientos sociales mantienen independencia a nivel organizativo y libertad general de acción. En cuarto lugar, una relación de independencia se da cuando los movimientos sociales actúan independientemente, presionando a los partidos para que se tomen medidas cercanas a su postura bajo la amenaza de que, al no hacerlo, podrían perder el apoyo de los simpatizantes del movimiento. Finalmente, existe una relación de competencia cuando una organización de movimiento social se transforma en un partido y pasa a competir en la arena electoral. Si aplicamos estas categorías a la realidad uruguaya, la historia reciente de los movimientos sociales en este respecto puede ser caracterizada como la de una alianza con el FA.

La victoria electoral del FA en el año 2004 generó un cambio importante en la estructura de oportunidad política del país. Los años de gobierno frenteamplista abrieron canales de comunicación inéditos para las organizaciones de movimientos sociales y se tradujeron en distintas políticas públicas y reformas legislativas que retomaron distintas demandas sociales: reinstauración de la negociación colectiva, ley de interrupción voluntaria del embarazo, mayor presupuesto para la educación, acciones afirmativas para la población afro y trans, matrimonio igualitario, avances en la lucha contra la impunidad de la violación de derechos humanos durante la dictadura etc. (Allier Montaño, 2015; Bidegain, 2013; Méndez; Senatore, 2013). 
Mientras que en muchos países de la región el giro a la izquierda tensionó fuertemente los vínculos entre la izquierda política y social que se habían forjado en la oposición a las reformas neoliberales de los noventa (Silva; Rossi, 2018), en Uruguay se mantuvo una tónica general y alianza durante los quince años, a pesar de algunas expresiones concretas de desencuentros (Moreira, 2010). En algunos casos, la cercanía entre los movimientos y el FA se tradujo en dinámicas propias al activismo burocrático, ya que se abrieron espacios institucionales en el Estado en que distintos activistas se incorporaron. El tipo de relacionamiento dependió de los distintos movimientos. Por ejemplo, mientras que el movimiento feminista tuvo un carácter multinodal que implicó, entre otras cosas, la inserción en espacios institucionales, el movimiento de la diversidad se mantuvo más orientado al campo social, a pesar de desarrollar algunas alianzas con actores estratégicos (Johnson et al., 2019, p. 49-50). En el caso de movimientos tradicionales, como el sindicalista, cooperativista o estudiantil, se tradujo en distintas políticas que atendieron reclamos históricos de estos actores y un vínculo menos conflictivo que el existente con los gobiernos anteriores, sin que se diera un alineamiento total de posiciones, ni una relación de articulación en términos de Hanagan. En muchos casos, los movimientos combinaron el uso de vínculos de cercanía con actores del FA con la presión en las calles para promover agendas que no eran de prioridad gubernamental pero que, por el tipo de temas, resonaban en el apoyo electoral de la coalición frenteamplista (Johnson et al., 2019).

En síntesis, si bien existieron algunos desencuentros en este período, estos no llegaron a cuestionar la histórica relación de colaboración entre los movimientos sociales y la coalición de izquierda uruguaya (Bidegain; Tricot, 2017). Si se retoman las categorías de relacionamiento entre partidos, gobiernos y movimientos en el giro a la izquierda propuestas por Bringel y Falero (2016), existieron relaciones negociadas y discontinuadas entre el gobierno del FA y sus tradicionales aliados, dependiendo de los casos.

Lo anterior no quiere decir que durante el ciclo progresista no hayan surgido conflictos y protestas por parte de movimientos que se posicionaron 
en franca oposición a la agenda frenteamplista. En las próximas secciones destacamos tres casos que dan cuenta de cambios en el patrón clásico de la conflictividad en Uruguay, especialmente en lo referido al vínculo con la izquierda política: el movimiento anti derechos, la ola de protestas del movimiento ambientalista uruguayo contra inversiones extranjeras directas, y el surgimiento del movimiento Un Solo Uruguay.

Es interesante destacar que algunas aristas de estos casos acercan a Uruguay a debates y experiencias que existen en el contexto latinoamericano, y que parecían, hasta este entonces, relativamente ajenas a la experiencia de este país. Particularmente, la presencia de grupos anti-derechos con un fuerte discurso conservador y religioso tiene una trayectoria más dilatada en otros países de la región y sorprendió recientemente en el escenario político uruguayo (Zaremberg et al., 2021). Además, la llegada de importantes inversiones extranjeras directas para proyectos con alto impacto a nivel ambiental ha sido foco de importantes conflictos en la región desde hace décadas. Uruguay ha sido un caso tardío en este sentido, pero, en los últimos años, surgieron conflictos que han llevado a los actores sociales involucrados a observar con atención lo que ha sucedido en la región y adoptar un discurso anti-extractivista (Santos, C., 2017).

Como será desarrollado, cada caso seleccionado tiene novedades y continuidades con la matriz histórica de protesta en el país. Para dar cuenta de esto, en cada uno de ellos presentaremos las siguientes dimensiones: demandas principales, características y repertorios de acción, y vínculos con los partidos.

\section{Nuevas conflictividades en el progresismo uruguayo}

\section{La reacción ante la nueva agenda de derechos}

SURGIMIENTO Y DEMANDAS

Uruguay ha sido un actor relevante en la marea rosa (Bull, 2013), introduciendo una nueva agenda de derechos (NAD) con énfasis en derechos sexuales y reproductivos y grupos históricamente oprimidos 
(Johnson et al., 2018). Los avances generaron una reacción social a esta agenda (Corrêa; Parker, 2020). En esta sección analizaremos a los grupos que, en contraposición a la NAD, han sido denominados como "anti-derechos". ${ }^{2}$

Inicialmente, a partir de la aprobación de la Ley de Interrupción Voluntaria del Embarazo, las demandas estuvieron primero canalizadas por grupos anti-aborto, de notoria carga religiosa, que luego migraron a concentraciones genéricas por "los valores" en los que se incluía la oposición a otras reformas de los gobiernos frenteamplistas, como el Matrimonio Igualitario, la regulación de la marihuana o la legislación orientada a reconocer derechos de las personas trans. Sin embargo, debido a que el framing de la NAD resultó indisputable, sus opositores tuvieron que adoptar una lógica discursiva extendida del paraguas conceptual de los derechos humanos (Puntigliano Casulo, 2020). Lo hicieron a través de lo que Wynn (2021) denomina como indirect bigotry: la "inocencia del gatekeeper" no necesariamente niega a las entidades sujetas de derechos, sino que entiende que se debe recortar su acceso a los derechos por entenderlos como aparentes privilegios frente a los derechos del resto.

De esta forma, el cuestionamiento específico alude a una crisis de valores en el marco de un aparente autoritarismo social y moralizante de la NAD (Entre, 2020; Torres Santana, 2019). Paradójicamente, la preocupación central apunta a la violación a la laicidad, en tanto la NAD ha permeado el Estado, presuntamente atentando contra la integridad de los niños, y, en especial, a cómo el efecto dañino del "lobby gay", según este discurso, promueve homosexualizar y hormonizar a los niños (Iglesias et al., 2020). Así, se camuflan en nombre de los derechos humanos, que presentándose como inclusivos buscan recortar derechos adquiridos (Torres Santana, 2019), secularizando su discurso bajo una "fachada plural y democrática" (Entre, 2019). Se propone el género como un elemento ideologizante a combatir, agrupando inquietudes sobre la seguridad ciudadana, la necesidad de la

${ }^{2}$ Aquí entendemos pertinente retomar la categoría nativa "movimientos sociales" ya que expresa el carácter reactivo del fenómeno a analizar. 
militarización y otras propuestas punitivas consideradas necesarias ante la "pérdida de valores" (Iglesias et al., 2020; Entre, 2019; Torres Santana, 2019).

\section{CARACTERÍSTICAS Y REPERTORIOS DE ACCIÓN}

Los grupos anti-derechos forman parte de un fenómeno transnacional con elevados recursos y bases solidificadas en organizaciones religiosas (Uval; Iglesias, 2020; Entre, 2019), especialmente encabezadas por evangelistas (Del Campo; Resina, 2020; Torres Santana, 2019), que, en los últimos años, ha volcado sus esfuerzos en América Latina.

Su objetivo central es "imponer una agenda política neoconservadora, en la que confluyen sectores católicos, evangélicos y agrupaciones políticas de extrema derecha" (Adrianzén; Yáñez, 2020, p. 40), emprendiendo la lucha contra una inventada ideología de género, retórica utilizada para deslegitimar a los movimientos feministas y de diversidad sexual (Garbagnoli, 2016). Excepcionalmente, Uruguay no tiene sus sustentos únicamente en la matriz religiosa, clivaje que históricamente no ha logrado trascender. Esto, sin embargo, no significa que la religión no haya tomado acción en contra de estos temas, pese a que Uruguay sea un Estado secular desde el temprano siglo XX (Johnson et al., 2018).

Pérez Bentancur y Rocha Carpiuc (2020) han creado una tipología útil para entender puntualmente el backlash en relación a estos temas mediante una matriz de doble entrada: por un lado, se pueden identificar estrategias institucionales (mecanismos formales) y no institucionales (informales y por lo general contenciosos) y, por otro lado, los distintos niveles macro (grupos organizados) y micro (individualizados) en los que operan los actores del backlash.

En la dimensión no institucional, los grupos anti-derechos hacen uso de sus redes transnacionales para conseguir e intercambiar elementos materiales, económicos, discursivos e informativos sobre posibles hojas de ruta creados por think tanks conservadores que aportan a cumplir su objetivo de combatir la ideología de género. Los slogans comunicacionales 
como \#ConMisHijosNoTeMetas sufren leves variaciones en la región y las herramientas audiovisuales utilizadas son idénticas en todos los países. La utilidad política de esto es demostrar la enorme capacidad organizativa que tienen, pero sobre todo que su voluntad de acción es difícilmente erosionable dados los recursos económicos de los que disponen. También ha quedado demostrado que, pese a no lograr manifestaciones masivas, a lo largo de los años han logrado movilizar una pequeña base social en reacción a episodios concretos. ${ }^{3}$

Estos grupos utilizan además la religiosidad como instrumento para reciclar el modelo de delegación de políticas públicas que el propio Estado era incapaz de sostener, mediante la presentación a licitaciones en las que si bien apuntan resolver asuntos específicos (como el consumo problemático de sustancias), transmiten también contenidos vinculantes a la agenda anti-género. ${ }^{4}$

En términos institucionales, también han tenido algún grado de incidencia. En el nivel micro se registraron varias organizaciones, de las cuales sólo pocas se han sostenido en el tiempo. ${ }^{5}$ Pero también en plataformas descentralizadas como el Congreso Nacional de Educación Pública del Sistema Nacional de Educación Pública se vio un involucramiento activo de sus demandas en contra de la educación sexual impartida por el Estado (SNEP, 2017). El nivel macro ha sido más evidente debido a la visibilidad pública que conlleva. Durante la discusión en comisiones parlamentarias, varias asociaciones ad hoc se presentaron para dar sus aportes sobre ambas normativas. Al fallar este mecanismo, juntaron firmas que habilitaron la

3 Por ejemplo, la Marcha por los Valores (10/11/2012), una concentración frente al MEC contra la Guía de Educación Sexual de Primaria (18/11/2017) y los intentos de contramarcha con carteles "Femeninas sí, feministas no" que se han registrado desde el 2017 en las Marchas del \#8M.

4 Existen, sin embargo, también experiencias positivas de licitaciones ganadas por organizaciones religiosas que trabajan con perspectiva de derechos humanos (Iglesias, 2013). ${ }^{5}$ La mayoría de éstas se proponían no explícitamente ir contra la ideología de género sino por contrario velar por los derechos de los niños (por nacer y nacidos), los valores de la familia y también otras nociones sin demasiada profundización como el "síndrome de alienación parental". Fenómenos como estos han sido estudiados por Iglesias et al. (2020) y cuentan con antecedentes teóricos en la teoría sobre el backlash de Susan Faludi. 
realización de pre-referéndums contra dos leyes específicas. El primero en contra de la Ley de Interrupción Voluntaria del Embarazo (2012) presentado por el diputado del PN Pablo Abdala junto al apoyo de coordinadoras ad hoc en contra del aborto, y segundo en contra de la Ley Integral para Personas Trans (2018), conducido por los autoproclamados líderes contra la NAD, Álvaro Dastugue y Carlos lafigliola (también del PN y con directa vinculación religiosa). Ambas iniciativas fracasaron al obtener solamente $8,92 \%$ y $9,91 \%$ de las adhesiones respectivamente.

\section{VÍNCULOS CON PARTIDOS POLÍTICOS}

Bottinelli, Selios y Bottinelli $(2017$, p. 2) proponen que "en los últimos cuarenta años las identidades políticas de los partidos tradicionales - PC y PN - han ido mutando hacia la consolidación de las llamadas familias ideológicas", donde algunos partidos se unen mediante la aproximación discursiva, la explícita diferenciación del progresista FA y sobre todo en el posicionamiento respecto a valores socioculturales, tales como la NAD (Bottinelli et al., 2017). Tomando las categorías de Hanagan (1998), los actores anti-derechos desarrollan vínculos con los sectores más conservadores del PC y, en especial, los sectores religiosos del PN, aunque de distinto tipo (Iglesias et al., 2020; Johnson et al., 2018).

Aprovechando el fenómeno de las familias ideológicas, la permeabilidad (Hanagan, 1998) es evidente cuando actores políticos evangelicalistas (Santos, B., 2014) tales como Álvaro Dastugue y Carlos lafigliola presentan propuestas que responden a su pertenencia orgánica religiosa, donde incluso el primero aludió a revisar y derogar la NAD en caso de que el PN llegara al gobierno nacional. Por otro lado, el sector de Verónica Alonso (PN) demuestra el tipo alianza (Hanagan, 1998) con la Iglesia Universal del Reino de Dios, del también evangelicalista pastor Jorge Márquez. Entonces, los grupos antiderechos logran vincularse con algunos sectores más conservadores de los partidos tradicionales, pero también han llegado a otros de más reciente creación como el nacional-militarista Cabildo Abierto, que en su programa de gobierno hace mención directa a la ideología de género. 
La síntesis central radica en aprovechar la escena de polarización en la que el FA es directamente asociado con la NAD, proponiendo así una idónea posición contraria de una alianza estrecha entre los anti-derechos y la derecha partidaria. Mientras, en particular en el PN, se apunta a movilizar su base electoral religiosa, en los demás partidos se busca llegar mediante un discurso generalizado de "lo que está bien versus lo que está mal", donde la imprecisión del enemigo es lo que facilita sus herramientas de coordinación (Entre, 2019). Sin embargo, estas plataformas partidarias no necesariamente logran siempre colmar los objetivos, mas resultan sin duda un instrumento útil como actores tomadores de decisiones anteriormente a nivel parlamentario, pero ahora también en el Ejecutivo.

\section{Las inversiones de gran porte y el movimiento ambiental}

\section{SURGIMIENTO Y DEMANDAS}

América Latina ha sido testigo en las últimas décadas de lo que ha sido denominado como el "Consenso de los commodities" que "apunta a subrayar el ingreso a un nuevo orden económico y político, sostenido por el boom de los precios internacionales de las materias primas y los bienes de consumo, demandados cada vez más por los países centrales y las potencias emergentes" (Svampa, 2012, p. 15). Como resultado de estos procesos, se ha señalado que la región ha consolidado un estilo de desarrollo extractivista (Gudynas, 2009).

Este modelo de desarrollo productivo ha permeado las economías de toda la región, independientemente de la orientación política de los gobiernos (Svampa, 2012). Uruguay no ha escapado de esta realidad. En este caso, uno de los principales proyectos enmarcados en este modelo de desarrollo productivo fue la proyección de una megaminería a cielo abierto en el país, interés que surgió por el alto precio del hierro en el mercado internacional. Este proyecto nace en el 2011 con la propuesta de inversión del grupo Zamin Ferrous, de capitales indios, con el fin de llevar adelante el Proyecto Aratirí. 
Aratirí fue un gran impulso para la movilización social: "el movimiento que se gestó con el proyecto de Aratirí (la mina, el mineroducto y el puerto), gestó por primera vez un movimiento que nosotros llamamos socioambiental en Uruguay. En otros países de la región no es novedad, pero en Uruguay nunca se había cristalizado." ${ }^{6}$ En este sentido, la principal demanda del movimiento estuvo centrada en que no se desarrollara en el país un proyecto minero como el que se proponía, y para esto utilizaron los medios a su alcance con el objetivo de cancelar la inversión.

Como consecuencia del avance de las inversiones, y con la demanda central de frenar los proyectos, la capacidad de organización de los colectivos fue aumentando y aglutinando diversas voces que, progresivamente, fueron ocupando la agenda ambiental en reacción a conflictos puntuales. ${ }^{7}$

\section{CARACTERÍSTICAS Y REPERTORIOS DE ACCIÓN}

El movimiento ambiental tiene características propias que lo distinguen de manera significativa del resto de los movimientos sociales en el país. Estas características están marcadas tanto por su organización y composición, así como por sus repertorios de acción (Elizalde et al., 2013).

En primer lugar, el movimiento cuenta con una integración heterogénea de actores, destacándose en dicha diversidad productores locales afectados por la mina, así como vecinos de la zona costera afectados por el puerto de aguas profundas proyectado para explotar el hierro, como también organizaciones de la sociedad civil que cuentan con una mayor capacidad técnica o también grupos de izquierda más radicales. Pese a esta heterogeneidad, que parte de intereses diversos y que se asocian a problemáticas particulares, el movimiento ha logrado construir un discurso marco común contra el modelo extractivista en el país (Bidegain; Freigedo, 2019).

En segundo lugar, los repertorios de acción rompieron con la clásica lógica centralista de la mayoría de los movimientos sociales, que centran

${ }^{6}$ Entrevista a miembro de la Asamblea Nacional Permanente.

${ }^{7}$ Si bien el proyecto Aratirí logró impulsar el movimiento, los actores también avanzaron en la agenda de demandas, oponiéndose a otros proyectos como la Ley de Riego, el cuidado de los Humedales del Río Santa Lucía, el Acuífero Guaraní, entre otros. 
sus protestas en Montevideo. En este sentido, el movimiento estructuró distintos niveles de acción, articulando las organizaciones territoriales a nivel local, con otras a nivel regional (como la Confederación de los Pueblo Costeros), y otras de nivel nacional (Asamblea Nacional Permanente o MOVUS).

En tercer lugar, y asociado a lo anterior, el movimiento tiene una lógica local y reactiva a las inversiones. Los actores se comenzaron a organizar localmente a partir del surgimiento de las inversiones privadas en cada lugar. Ejemplos claros de esta lógica reactiva son el caso de Valentines, donde los productores rurales se organizaron ante la construcción de la minera en ese territorio, o el caso de los residentes de la zona costera de Rocha ante la construcción de un puerto de aguas profundas que permitiera la salida de la producción minera.

Por último, en relación a los repertorios de acción, el movimiento ambiental ha utilizado diferentes mecanismos para confrontar los proyectos. Por un lado, se han utilizado los clásicos mecanismos de manifestaciones en espacios públicos, tanto a nivel local como a nivel nacional, promoviendo anualmente en la capital del país la "Marcha Nacional en Defensa de la Tierra, el Agua y la Vida", convocada por la ANP. Por otro lado, también se han utilizado recursos institucionales de democracia directa para manifestar sus demandas. En este sentido, es especialmente relevante destacar la promoción de recolección de firmas para someter a referéndum nacional la posibilidad de promover proyectos de minería a cielo abierto en el país, así como la recolección de firmas para derogar la Ley de Riego ${ }^{8}$. Incluso, en algunos casos a nivel local se juntaron firmas para declarar los territorios libres de minería y "fracking". 9

\footnotetext{
${ }^{8}$ Vale decir que en ambos casos no se llegaron a las firmas necesarias para promover los mecanismos de democracia directa.

${ }^{9}$ En el caso del Departamento de Tacuarembó, si bien se alcanzaron las firmas necesarias y el tema fue abordado por el gobierno departamental (segundo nivel) con una resolución que prohibía en el Departamento la Minería a cielo abierto y el Fracking -, desde el nivel central se interpuso un recurso administrativo con el argumento de que no es potestad de los gobiernos subnacionales decidir sobre el uso del subsuelo en el territorio.
} 
EL MOVIMIENTO AMBIENTAL Y EL VÍNCULO CON EL SISTEMA POLÍTICO

El movimiento ambiental ha tenido a lo largo de estos años un relacionamiento de desconfianza con todos los partidos políticos. Como expresa un miembro de la Asamblea Nacional Permanente en Defensa de la Tierra y los Bienes Naturales (ANP): "desde sus orígenes, la ANP se define como apolítica. No funcionará con la política partidista. Las banderas partidistas no están permitidas ni en las manifestaciones ni en las asambleas. Las personas con afinidades partidistas son bienvenidas, pero las organizaciones políticas no lo son". Por tanto, siguiendo las categorías de Hanagan (1988), es posible afirmar que el movimiento ha entablado un vínculo de independencia con los partidos políticos.

De todas formas, algunas organizaciones buscaron llegar a ciertas estructuras locales del FA con el fin de encontrar aliados desde las bases militantes. De hecho, se mantuvieron encuentros en los Comités de Bases frenteamplistas con el objetivo de presentar directamente a los militantes del partido sus ideas sobre Aratirí y sus proyectos relacionados. Sin embargo, en algún momento estas experiencias fueron suspendidas desde las estructuras partidarias. De acuerdo a algunos entrevistados, hubo presiones desde el nivel central del partido que truncaron la posibilidad de continuar con este tipo de encuentros.

Esta independencia, y el relacionamiento distante del movimiento ambiental con los partidos políticos, también se reprodujeron con otros actores sociales, particularmente con el movimiento sindical. En este sentido, si bien el apoyo del movimiento sindical se podría transformar en un aliado fundamental para cumplir con sus objetivos, y se hicieron esfuerzos para lograrlo, los resultados fueron negativos. Según un entrevistado, la dinámica que existió con el FA se replicó con la central sindical uruguaya: "lo mismo ocurrió, creo, con muchos sindicatos. Sí, digamos que al principio hubo un acercamiento, un entendimiento, al menos un Ilamado al diálogo. Pero la misma dinámica se repitió: el PIT-CNT llegó, las conversaciones 
disminuyeron, y luego un cierre total. Se acabó." El movimiento sindical apoyó los proyectos relacionados a Aratirí bajo el argumento fundamental de las fuentes laborales asociadas a las inversiones.

\section{Un Solo Uruguay}

SURGIMIENTO Y DEMANDAS

Uruguay se ha caracterizado históricamente por ser un país agroexportador, donde los sectores rurales han tenido un peso importante en la vida política nacional, sobre todo a partir de la acción de sus asociaciones gremiales. ${ }^{10}$ Sin embargo, en el último período de gobierno frenteamplista surgió el movimiento denominado "Un Solo Uruguay", que rompió con la lógica clásica de intermediación entre los sectores rurales y el sistema político nacional.

Las demandas del movimiento surgieron como consecuencia de las transformaciones en la estructura de producción agropecuaria clásicas en Uruguay. A partir de inversiones de grandes corporaciones multinacionales agroexportadoras se ha modificado el tipo de producción hacia nuevos sectores como el sojero. Esto ha derivado en una mayor dependencia del sector agroexportador al comportamiento del mercado y particularmente al precio internacional de las commodities.

Si bien durante la primera década de los gobiernos de izquierda los precios estuvieron al alza, a partir de 2014 el ciclo de auge de los precios de los productos agropecuarios se terminó, y eso derivó en tensiones distributivas entre diferentes sectores, sobre todo a partir de la pérdida de rentabilidad de algunos eslabones de la cadena de valor agroindustrial (Monestier, 2019).

Esta tensión generó diversas reacciones, sobre todo de productores locales que comenzaron a manifestarse de manera esporádica y desorganizada, generalmente a los costados de las rutas nacionales.

${ }^{10}$ Asociación Rural del Uruguay (ARU), Federación Rural (FR). 
Dichas manifestaciones comenzaron a encontrar aliados a nivel local de algunas asociaciones departamentales de comerciantes, transportistas o rematadores, lo que derivó en una primera gran concentración de lo que ellos mismos denominaron como "auto convocados" en el Departamos de Durazno ${ }^{11}$ (Piñeiro; Cardeillac, 2018). Este fue el hito más importante del movimiento, que logró nuclear entre 50.000 y 60.000 personas dependiendo de las estimaciones. ${ }^{12}$

En estos primeros encuentros, se plantearon las principales demandas que han llevado adelante la agenda del movimiento. Principalmente, su mayor objetivo pasa por el excesivo peso del Estado en la economía y el aumento del gasto público. Por tanto, los planteos están asociados a cuestiones relacionadas a reducir el gasto público y la carga impositiva al sector ganadero e industrial.

\section{CARACTERÍSTICAS DEL MOVIMIENTO Y REPERTORIOS DE ACCIÓN}

El movimiento "Un solo Uruguay" se define como:

Una organización de alcance nacional que representa a trabajadores y productores de las áreas de la economía nacional que dinamizan y dan vida al país: el sector agropecuario en toda su extensión, el comercio, el sector empresarial, los servicios, el turismo, los profesionales. Surgimos en el interior del país, desde el empuje en conjunto de decenas de organizaciones y asociaciones que vieron la necesidad de crear un nuevo movimiento nacional. ${ }^{13}$

En este sentido, un primer punto a destacar es la heterogeneidad del movimiento. Si bien al inicio los principales actores eran productores ganaderos, a medida que las protestas se extendieron, el movimiento logró captar la atención de otros sectores de la economía, incluso muchos

\footnotetext{
${ }^{11}$ Este Departamento se encuentra en el centro del país, punto neurálgico para que pudieran llegar desde diferentes puntos del territorio.

${ }^{12}$ Según La Diaria (24/01/18), 50.000 personas, y 60.000 personas según El País (sección Rurales, 24/01/18).

13 Información disponible en: https://seragro.com.uy/portada/usu-y-sus-criticas-al-sistemapolitico-al-gobierno-anterior-y-al-actual/
} 
de ellos que estaban radicados en la capital del país. Un estudio sobre las características de los voceros del movimiento en cada uno de los 19 Departamentos del país da cuenta de la diversidad. ${ }^{14}$ En este sentido, los productores agropecuarios suman sólo 53\% de los voceros del movimiento. Los profesionales son $17 \%$, mientras que los empresarios y comerciantes son otro $10 \%$. Los empresarios de servicios ligados al agro representan el $17 \%$.

Un segundo aspecto que caracteriza al movimiento es su lógica descentralizada, ya que, si bien, como se mencionó, el movimiento tuvo sus orígenes en el sector rural, rápidamente ha captado la adhesión de diferentes actores, generando una estructura de representación en todos los departamentos del país.

Un tercer aspecto tiene que ver con el tipo de demandas que plantea el movimiento. Estas parten de un marco común que permite tomar en cuenta la diversidad de actores que lo integran, superando únicamente al sector rural. Como se mencionó, estas demandas están asociadas a la pesada carga de la estructura estatal.

En relación a los repertorios de acción, el movimiento ha combinado diversas estrategias. En primer lugar, los encuentros como la concentración mencionada de Durazno, en los cuales la lectura de una proclama es el punto central. En segundo lugar, han marchado y manifestado frente a instituciones públicas. Por último, también han utilizado mecanismos de democracia directa. Específicamente han buscado recolectar firmas en contra de una nueva planta de fabricación de celulosa en el país, que se basa en grandes beneficios fiscales, lo que ilustra la puja distributiva del sector (Monestier, 2019). Apoyaron también otras dos iniciativas en este sentido. Por un lado, una iniciativa que buscó flexibilizar las condiciones de contratación de los trabajadores rurales, por medio de la eliminación de normas que regularizan el trabajo formal (Monestier, 2019). Por otro lado, apoyaron la recolección de firmas para eliminar la Ley de Inclusión

${ }^{14}$ Informe disponible en: https://ladiaria.com.uy/articulo/2018/3/ocupaciones-y-posicionespoliticas-de-los-voceros-de-los-autoconvocados-en-los-19-departamentos/ 
Financiera, que plantea regular los medios de pago electrónicos buscando formalizar la economía. Desde el movimiento, esto se veía como una traba para la independencia financiera del sector. En todos los casos no se alcanzaron los resultados deseados.

\section{Un Solo Uruguay y el Vínculo con el sistema político}

Como se mencionó, la incorporación de "Un solo Uruguay" modificó la lógica de intermediación política entre los sectores rurales y los partidos políticos. Hasta ese momento, la lógica corporativa primaba, y el relacionamiento, incluso con los gobiernos de izquierda, se basaba en encuentros informales entre jerarcas de las principales instituciones gremiales y las autoridades de gobierno.

Por tanto, la irrupción del movimiento implicó un factor de inestabilidad, no solo para el gobierno sino también para los actores gremiales clásicos, que quedaron en la encrucijada de apoyar al nuevo movimiento, pero buscando no perder su capacidad de acción ante el gobierno. De todas formas, la estrategia del movimiento fue mantenerse como aliado de las organizaciones clásicas, sin disputar los espacios, pero dejando en claro su autonomía (Monestier, 2019).

En cuanto al vínculo del movimiento con los partidos políticos, desde la primera proclama se manifestaron independientes de todos los partidos. De todas formas, parece claro que la agenda del movimiento tiene muchos puntos de encuentro con los planteados por los partidos políticos que en ese momento eran oposición, fundamentalmente en lo que respecta al gasto público y la reducción del costo del Estado. En este sentido, un alto jerarca del gobierno frenteamplista manifestaba lo siguiente: "(...) cuando "Un solo Uruguay" hizo avanzar al campo atrás de un proyecto, era el proyecto de la oposición (...) ellos plantearon el programa que tienen los partidos tradicionales". ${ }^{15}$

${ }^{15}$ Disponbile en: http://radiouruguay.uy/bonomi-un-solo-uruguay-esta-atras-del-programade-los-partidos-tradicionales 
Por tanto, siguiendo las categorías de Hanagan (1988), si bien desde el movimiento se plantea la independencia total, los intereses comunes sobre la agenda particular en cuanto al rol del Estado, pueden significar una alianza ad hoc.

\section{Consideraciones finales}

Durante el ciclo progresista del FA (2005-2020) se dio una dinámica de conflictividad sociopolítica que significó continuidades y cambios con respecto a la experiencia de las dos décadas anteriores. En los párrafos siguientes sintetizamos los principales aspectos de este período y presentamos algunas reflexiones sobre el escenario que se abre con el retorno de los partidos de derecha al poder en 2020.

Primero, es importante destacar que no existieron en estos quince años episodios de revuelta social que pusieran en riesgo la estabilidad política del país o demandaran cambios sustantivos en sus premisas institucionales básicas. Esta es una línea de largo alcance que caracteriza al país, y que ha sido señalada por estudios comparados regionales (Joignant et al., 2017). En términos generales, y en concordancia con la experiencia anterior del país, se mantuvo una dinámica de conflictividad social orientada a temas específicos, con una centralidad importante del sistema de partidos como canalizador de demandas, y el uso en distintos casos de los mecanismos de democracia directa por parte de actores sociales y políticos para buscar impactar en el marco normativo del país.

En este sentido, Uruguay mantiene ciertas peculiaridades en su sistema institucional (existencia de mecanismos de democracia directa vinculantes, por ejemplo) y de su sistema de partidos (altamente institucionalizado, y central en la dinámica política y social del país) que vuelven particularmente útil un enfoque teórico y metodológico preocupado por los vínculos entre política institucional y no institucional como el desarrollado en este artículo. No obstante, la emergencia de actores y discursos como el anti-extractivista, que cuestionan al sistema partidario en su conjunto, llama a cuestionarse 
sobre otros enfoques teóricos que puedan captar este tipo de dinámicas. En futuros trabajos, sería importante avanzar en la comparación con otros casos latinoamericanos, de forma de identificar otras perspectivas que puedan complementar el análisis.

En segundo lugar, a diferencia de los movimientos sociales históricos del campo popular uruguayo (trabajadores, cooperativista, estudiantil etc.) y de los promotores de la NAD (feminista, diversidad etc.), los casos que aquí se analizaron no tuvieron vínculos cercanos con la izquierda en el gobierno. Por el contrario, su posicionamiento marcó distancias con el partido gobernante y apuntó en primera instancia a sostener demandas tanto propositivas como reactivas que serían incompatibles con la agenda del FA, incluso cuando casos como el ambientalista tienen vínculos con la izquierda social. Esta forma de relacionamiento con el sistema político inevitablemente resultó en que algunas de estas acciones colectivas tuvieran mayor eco en partidos de derecha, que se amoldaron a sus demandas, no solamente en términos de contenidos, sino por lograr conformar una familia ideológica que continuamente interpelara al FA.

En tercer lugar, han surgido demandas que han llevado a la aparición de nuevos movimientos sociales o a la reconfiguración de algunos de ellos. En este sentido, si bien, como se mencionó, existieron otros tipos de demandas, este artículo se centró en dos asuntos fundamentales. Por un lado, las nuevas inversiones y formas de producción en el territorio han llevado a la movilización de dos colectivos con intereses diferentes: el movimiento ambiental y Un Solo Uruguay. El movimiento ambiental, si bien no es un actor novedoso, ganó mucha fuerza a partir de la campaña contra el proyecto minero de Aratirí, movilizándose con el objetivo de cancelar la inversión. Por su parte, Un Solo Uruguay surge como consecuencia de la dependencia de la economía agroexportadora de los precios hacia las materias primas. La caída de los precios internacionales llevó a que diversos actores de la cadena productiva ser vieran afectados se movilizaran para 
hacer sentir sus demandas en la puja distributiva. Por otro lado, el avance de la NAD llevó al surgimiento de un movimiento que se opuso a estas políticas a través del uso de los mecanismos de democracia directa y la apelación a un estilo discursivo basado en una retórica de derechos humanos para presentar su desacuerdo ante un consenso social instalado.

En cuarto lugar, las características que han asumido los conflictos analizados también presentan algunas novedades particulares. Por una parte, destaca que la lógica territorial de las inversiones ha llevado a que también los movimientos tengan una presencia en el territorio que no es habitual en el país. La lógica descentralizada de los actores colectivos (tanto ambiental como de Un Solo Uruguay) rompe con el centralismo clásico de los movimientos sociales uruguayos, basado en Montevideo. Por otra parte, en el caso de la reacción a la NAD, la acción colectiva es especialmente dependiente de su marco de alianzas internacional, tanto económicamente como en sus contenidos.

En quinta instancia, en relación a los repertorios de acción, una interesante novedad del período analizado fue el uso de los mecanismos de democracia directa por parte de actores que tradicionalmente no habían utilizado estas herramientas. En todos los conflictos analizados, los actores colectivos han impulsado o apoyado estos mecanismos, pese a que luego fallaran electoralmente. En ningún caso las iniciativas lograron un apoyo mayoritario que alterara las decisiones de política. Esto tiene que ver posiblemente con los escasos apoyos que han tenido de los movimientos sociales uruguayos clásicos, así como del resto del sistema político, particularmente de los partidos políticos. También está probablemente vinculado a la incapacidad de generar una hegemonía sociocultural que habilitara una transformación estructural a partir de sus demandas.

Por último, respecto al vínculo con el sistema político, particularmente los partidos, es posible advertir diferentes niveles de relacionamiento según el tipo de movimiento social. En este sentido, el único movimiento que parece completamente independiente de los partidos políticos es el 
ambiental. En el resto de los casos, en mayor o menor medida, se identifican algunos vínculos asociativos con los partidos de derecha. Este patrón sigue la línea histórica de los movimientos sociales en Uruguay, que da cuenta de la importancia de los partidos para operar como canales privilegiados de intermediación con la sociedad civil.

Dicho esto, y dado que un nuevo escenario político con una coalición opositora al FA en el gobierno desde marzo de 2020, se plantean una serie de interrogantes. En primer lugar, será interesante observar si el movimiento ambientalista mantiene su tónica de independencia de todos los partidos políticos o si, al encontrarse el FA en la oposición, existe un acercamiento con la izquierda política del país. Durante el período 19852005 el FA demostró ser un aliado poderoso de los movimientos sociales desde la oposición. Por lo tanto, será importante ver si en el futuro se producen cambios en los conflictivos patrones de relacionamiento de este movimiento con el FA que se dieron durante los gobiernos progresistas. En segundo lugar, el acceso de la coalición de derecha al gobierno motiva a preguntarse si Un Solo Uruguay mantendrá su perfil de protestas públicas o si los sectores agrarios y empresariales retoman los canales clásicos de intermediación de intereses a través del lobby de las cámaras y asociaciones que los representan. Finalmente, una incógnita relevante refiere a las estrategias y capacidades de los grupos opuestos a la NAD para modificar el marco legal impulsado durante los gobiernos frenteamplistas y/o las orientaciones políticas de las actuales autoridades. Durante la campaña electoral de 2019, el presidente electo indicó su compromiso de no generar cambios normativos. No obstante, existen márgenes de acción relevantes en la aplicación de las políticas gubernamentales para aplicar el marco legal vigente que seguramente serán campo de disputa durante los años que restan del actual gobierno. 
Germán Bidegain es Doctor en Ciencia Política por la Pontificia Universidad Católica de Chile. Actualmente es profesor adjunto del Departamento de Ciencia Política de la Universidad de la República de Uruguay e investigador asociado del Centro de Informaciones y Estudios del Uruguay (CIESU). Es investigador nivel 1 del Sistema Nacional de Investigadores del Uruguay.

$\bowtie$ german.bidegain@cienciassociales.edu.uy

Martín Freigedo es Doctor en Ciencias Sociales por FLACSO-México. Actualmente es profesor adjunto del Departamento de Ciencia Política de la Universidad de la República. Es investigador nivel 1 del Sistema Nacional de Investigadores del Uruguay.

$\bowtie$ martin.freigedo@cienciassociales.edu.uy

Diego Puntigliano Casulo es Licenciado en Ciencia Política por la Universidad de la República y Diplomado en Promoción y Gestión de Derechos Culturales por la Universidad de Buenos Aires. Actualmente es estudiante de la Maestría Bimodal en Estudios Contemporáneos de América Latina de la Universidad de la República y la Universidad Complutense de Madrid, y es integrante del grupo de investigación-acción-participativa Área Académica Queer.

$\triangle \underline{\text { d.puntigliano@gmail.com }}$

\section{Referencias}

1. ABERS, Rebecca. Bureaucratic activism: pursuing environmentalism inside the Brazilian State. Latin American Politics and Society, v. 61, n. 2, p. 21-44, 2019. https://doi.org/10.1017/lap.2018.75

2. ABERS, Rebecca; VON BÜLOW, Marisa. Movimentos sociais na teoria e na prática: como estudar o ativismo através da fronteira entre estado e sociedade? Sociologias, v. 13, n. 28, p. 52-84, 2011. https://doi.org/10.1590/S1517$\underline{45222011000300004}$

3. ADRIANZÉN, Alberto; YÁÑEZ, Luis. Comunicar para adoctrinar: control de medios por grupos antiderechos en Perú, México y Costa Rica. In: CHAHER, Sandra (Comp.). Comunicación, Feminismo y Religión en América Latina. Ciudad de Buenos Aires: Asociación Civil Comunicación para la Igualdad Ediciones/FES Comunicación, 2020. p. 46-59.

4. ALLIER MONTAÑO, Eugenia. De historias y memorias sobre el pasado reciente en Uruguay: treinta años de debates. Caravelle. Cahiers du monde hispanique et luso-brésilien, n. 104, p. 133-150, 2015. https://doi.org/10.4000/caravelle.1615

5. ALTMAN, David. Direct democracy worldwide. Cambridge: Cambridge University Press, 2011.

6. BIDEGAIN, Germán. Uruguay, ¿el año bisagra? Revista de Ciencia Política, [s. I.], v. 33, n. 1, p. 351-374, 2013. 
7. BIDEGAIN, Germán; FREIGEDO, Martín. The La Paloma deepwater port project in Uruguay: local and national environmental resistance to the governmental agenda. In: 15 CONGRÈS DE L'ASSOCIATION FRANÇAISE DE SCIENCE POLITIQUE, 2019, Bordeaux, France. Anais [...]. Bordeaux, France: [s. n.], 2019.

8. BIDEGAIN, Germán; TRICOT, Víctor. Political Opportunity Structure, Social Movements, and Malaise in Representation in Uruguay, 1985-2014. In: JOIGNANT, Alfredo; MORALES, Mauricio; FUENTES, Claudio (Eds.). Malaise in Representation in Latin American Countries. Chile, Argentina, and Uruguay. New York: Palgrave Macmillan, 2017. p. 139-160.

9. BOTTINELLI, Oscar; SELIOS, Lucía; BOTTINELLI, Eduardo. Espacios de competencia y significados de la ideología de izquierda y derecha en Uruguay. In: CONGRESO LATINOAMERICANO DE CIENCIA POLÍTICA, 9., 2017, Montevideo. Anales [...]. Montevideo: ALACIP, 2017.

10. BRINGEL, Breno; FALERO, Alfredo. Movimientos sociales, gobiernos progresistas y Estado en América Latina: transiciones, conflictos y mediaciones. Caderno CRH, v. 29, p. 27-45, 2016.

11. BULL, Benedicte. Social movements and the "pink tide" governments in Latin America: transformation, inclusion and rejection. In: STOKKE, Kristian; TÖRNQUIST, Olle (Eds.). Democratization in the global south: the importance of transformative politics. Londres: Palgrave Macmillan, 2013. p. 75-99. (International Political Economy Series).

12. CHASQUETTI, Daniel; BUQUET, Daniel. La democracia en Uruguay: una partidocracia de consenso. Política, v. 42, p. 221-247, 2004.

13. CORRÊA, Sonia; PARKER, Richard. Prefacio. In: IGLESIAS, Nicolás et al. (Eds.). Políticas antigénero en Latinoamérica. Uruguay, el mal ejemplo. Rio de Janeiro: Observatorio de Sexualidad y Política, 2020. E-book.

14. DEL CAMPO, María E.; RESINA, Jorge. ¿De movimientos religiosos a organizaciones políticas? La relevancia política del evangelismo en América Latina 2020. Documentos de Trabajo Fundación Carolina, v. 35, 2020.

15. DELLA PORTA, Donatella; DIANI, Mario. Los movimientos sociales. Madrid: Centro de Investigaciones Sociológicas - Universidad Complutense de Madrid, 2011.

16. ELIZALDE, Lucía; MENÉNDEZ, Mariana; SOSA, María. Cronología sobre el conflicto minero en Úruguay 2010-2013. Revista Contrapunto, v. 2, p. 47-71, 2013.

17. ENTRE (Org.). La reacción: derecha e incorrección política en Uruguay. Montevideo: Estuario Editora, 2019.

18. FALERO, Alfredo; FRY, Mariana. ¿Qué nos dicen los conflictos por la educación en el Uruguay? Una propuesta de análisis. Montevideo. In: CONGRESO ALAS, XXXI., 2017, Montevideo. Anales [...]. Montevideo: ALAS, 2017.

19. FILGUEIRA, Carlos H. Movimientos sociales en la restauración del orden democrático: Uruguay 1985. In: FILGUEIRA, Carlos H. (org.). Movimientos Sociales en el Uruguay de Hoy. Montevideo: Clacso/Ciesu/Ediciones de la Banda Oriental, 1985. p. 11-50. 
20. FILGUEIRA, Fernando et al. Los dos ciclos del Estado uruguayo en el siglo XX. In: NAHÚM, Benjamín; CAETANO, Gerardo (org.). El Uruguay del siglo XX. Tomo II. La política. Montevideo: Ediciones de la Banda Oriental - Instituto de Ciencia Política, 2003. p. 173-204.

21. GARBAGNOLI, S. Against the heresy of immanence: vatican's "gender" as a new rhetorical device against the denaturalization of the sexual order. Religion and Gender, v. 6, n. 2, p. 187-204, 2016.

22. GOLDSTONE, Jack A. States, parties, and social movements. New York: Cambridge University Press, 2003.

23. GUDYNAS, Eduardo. Diez tesis urgentes sobre el nuevo extractivismo. Contextos y demandas bajo el progresismo sudamericano actual. In: CAAP; CLAES. Extractivismo, política y sociedad. Quito: CAAP/CLAES, 2009.

24. HANAGAN, Michael. Social movements. Incorporation, disengagement, and opportunities - a long view. In: GIUGNI, Marco G.; MCADAM, Doug; TILLY, Charles. From contention to democracy. Lanham: Rowman \& Littlefield Publishers, 1998. p. 4-30.

25. JOHNSON, Niki; RODRÍGUEZ GUSTÁ, Ana Laura; SEMPOL, Diego. Explaining advances and drawbacks in women's and LGBTIQ rights in Uruguay: multisited pressures, political resistance, and structural inertias. In: FRIEDMAN, Elisabeth J. (org.). Seeking rights from the left: gender, sexuality, and the Latin American pink tide. Durham: Duke University Press, 2019. p. 48-81.

26. JOIGNANT, Alfredo; MORALES, Mauricio; FUENTES, Claudio (org.). Malaise in representation in Latin American countries. Chile, Argentina, and Uruguay. Nueva York: Palgrave Macmillan, 2017.

27. IGLESIAS, Nicolás. Religión y participación social. Montevideo: Ministerio de Desarrollo Social, 2013. (Serie Hablando de derechos | DESC+A, n. 22).

28. IGLESIAS, Nicolás; KREHER, Stefanie; ABRACINSKAS, Lilián; PUYOL, Santiago. Políticas antigénero en Latinoamérica. Uruguay, el mal ejemplo. Rio de Janeiro: Observatorio de Sexualidad y Política, 2020. E-book.

29. MCADAM, Doug. Political process and the development of black insurgency, 1930-1970. Chicago: University of Chicago Press, 1999.

30. MCADAM, Doug; MCCARTHY, John D.; ZALD, Mayer N. (org.). Comparative perspectives on social movements: political opportunities, mobilizing structures, and cultural framings. 1. ed. Cambridge: Cambridge University Press, 1996.

31. MCADAM, Doug; TARROW, Sidney; TILLY, Charles. Dynamics of contention. Cambridge: Cambridge University Press, 2001.

32. MÉNDEZ, Gustavo; SENATORE, Luis. El sindicalismo ante los gobiernos de la izquierda. Uruguay 2005-2010. In: ABAL MEDINA, Paula; FORNILLO, Bruno; WYCZYKIER, Gabriela. La forma sindical en Latinoamérica. Miradas contemporáneas. Buenos Aires: Nueva Trilce, 2013. p. 154-196. 
33. MONESTIER, Felipe. Conflictos distributivos, corporaciones agrarias y gobiernos de izquierda en Uruguay. El caso del movimiento "Un Solo Uruguay". In: SEMINARIO INTERNO DEL INSTITUTO DE CIENCIA POLÍTICA, 2019, Montevideo. Anales [...]. Montevideo: Universidad de la República, 2019.

34. MONESTIER, Felipe. Movimientos sociales, partidos políticos y democracia directa "desde abajo" en Uruguay (1985-2004). Buenos Aires: CLACSO, 2010.

35. MOREIRA, Carlos. Movimientos populares y luchas sociales en Uruguay. Interseções, v. 12, n. 2, p. 283-300, 2010.

36. MOREIRA, Constanza. Resistencia política y ciudadanía: plebiscitos y referéndums en el Uruguay de los 90. América Latina Hoy, v. 36, p. 17-45, 2004.

37. PÉREZ BENTANCUR, Verónica; ROCHA-CARPIUC, Cecilia. The postreform stage: understanding backlash against sexual policies in Latin America. Politics \& Gender, v. 16, n. especial 1, p. 11-18, 2020.

38. PIÑEIRO, Diego; CARDEILLAC, Joaquín. Where have all the flowers gone? The end of political consensus in the countryside. In: ANNUAL CONFERENCE IN POLITICAL ECONOMY, 9. 2018, Pula. Anales [...]. Pula: International Initiative for Promoting Political Economy, 2018.

39. PUNTIGLIANO CASULO, Diego. Militancia y (nuevas) derechas sexuales: politización de la diversidad sexual en los Partidos Colorado y Nacional. pp. 106, 2020. Disertación de grado (Licenciatura en Ciencia Política) - Facultad de Ciencias Sociales, Universidad de la República, Montevideo, 2020.

40. SANTOS, Boaventura de S. Si Dios fuese un activista de los derechos humanos. Madrid: Trotta, 2014.

41. SANTOS, Carlos. Los conflictos ambientales en el progresismo uruguayo. In: Jornadas de Investigación, XVI., 2017, Montevideo. Anales [...]. Montevideo: Universidad de la República, 2017.

42. SILVA, Eduardo; ROSSI, Federico. Reshaping the political arena in Latin America: from resisting neoliberalism to the second incorporation. Pittsburgh: University of Pittsburgh Press, 2018.

43. SILVA, Marcelo K.; OLIVEIRA, Gerson de L. A face oculta(da) dos movimentos sociais: trânsito institucional e intersecção Estado-Movimento - uma análise do movimento de economia solidária no Rio Grande do Sul. Sociologias, v. 13, n. 28, p. 86-124, 2011. https://doi.org/10.1590/S1517-45222011000300005

44. SNEP - Sistema Nacional de Educación Pública. Sistematización de opiniones y propuestas a nivel nacional. III Congreso Nacional de Educación Enriqueta Compte y Riqué, 2017. Disponible en: https://www.dgeip.edu.uy/documentos/2017/ varios/1974/congreso-de-educacion-2017.pdf

45. SVAMPA, Maristella. Consenso de los commodities, giro ecoterritorial y pensamiento crítico en América. Revista del Observatorio Social de América Latina, v. 32, p. 15-38, 2012. 
46. TORRES SANTANA, Ailynn. De la marea rosa a la marea conservadora y autoritaria en América Latina: desafíos feministas. Quito: Friedrich-Ebert-Stiftung (FES-ILDIS), 2019.

47. TILLY, Charles. From mobilization to revolution. Reading: Addison-Wesley, 1978.

48. UVAL, Natalia; IGLESIAS, Nicolás. Del "comunismo" a los feminismos: "de la marea roja a la marea rosa". La Diaria, p. 13-14, 21 nov. 2020. Disponible en: $\quad$ https://ladiaria.com.uy/politica/articulo/2020/11/del-comunismo-a-losfeminismos-de-la-marea-roja-a-la-marea-rosa/

49. VON BÜLOW, Marisa; BIDEGAIN, Germán. Se necesitan dos para bailar tango: estudiantes, partidos políticos y protesta en Chile, 2005-2013. In: ALMEIDA, Paul; CORDERO ULLAT, Allen (Eds.). Movimientos sociales en América Latina. Perspectivas, tendencias y casos. Ciudad Autónoma de Buenos Aires: CLACSO, 2017. p. 313-340,

50. WYNN, Natalie. J.K. Rowling | ContraPoints. Youtube, 26 jan. 2021. Disponible en: https://youtu.be/7gDKbT_Lus

51. ZAREMBERG, Gisela; TABBUSH, Constanza; FRIEDMAN, Elisabeth J. Feminism(s) and anti-gender backlash: lessons from Latin America. International Feminist Journal of Politics, v. 23, n. 4, p. 527-534, 2021. 